Proceedings of the 43rd IEEE Conference on Decision and Control

4818-4823-6089, December 2004

DOI: $10.1109 /$ CDC.2004.1429552

2004 IEEE. Personal use of this material is permitted. However, permission to reprint/republish this material for advertising or promotional purposes or for creating new collective works for resale or redistribution to servers or lists, or to reuse any copyrighted component of this work in other works must be obtained from the IEEE

\title{
Notes on energy shaping
}

Andrew D. Lewis*

$2004 / 03 / 30$

Last updated: $2004 / 12 / 04$

\begin{abstract}
The problem of shaping the kinetic and potential energy of a mechanical system by feedback is cast in a differential geometric framework. The nature of the set of solutions to the potential energy shaping problem is described. The kinetic energy shaping problem is posed in (1) an affine differential geometric framework and (2) a manner where the geometric integrability theory for partial differential equations can be applied.
\end{abstract}

\section{Introduction}

In recent years, there has appeared in the literature a significant number of publications on the subject of energy shaping. The procedure is, roughly, one wherein one alters by feedback a mechanical system in such a way that the closed-loop system is also mechanical. The principal advantage in doing this is that the stability of equilibria for simple mechanical systems, i.e., those with kinetic minus potential Lagrangians, is quite well understood. One can, in principle, use this understanding to design the closed-loop system to stabilise an equilibrium configuration, hopefully with a larger basin of attraction than would be possible via, for example, linearisation.

The first paper to consider the problem seems to be that of Takegaki and Arimoto [1981], where potential shaping was considered for systems with integrable input codistributions. A geometric treatment of potential shaping for systems with possibly nonintegrable input codistributions was given by van der Schaft [1986]. Other early work, in a Hamiltonian setting, is [Bloch, Krishnaprasad, Marsden, and Sánchez de Alvarez 1992]. Various groups have made recent contributions to this problem, notably by additionally considering kinetic energy shaping. The "method of controlled Lagrangians" is described in the papers [Bloch, Chang, Leonard, and Marsden 2001, Bloch, Leonard, and Marsden 2000]. The "interconnection and damping assignment-passivity based control (IDA-PBC) method" is described in [Ortega, Spong, Gómez-Estern, and Blankenstein 2002]. The two methods are (essentially) shown to be equivalent in the papers [Blankenstein, Ortega, and van der Schaft 2002, Chang, Bloch, Leonard, Marsden, and Woolsey 2002]. Both of the methods produce systems of nonlinear partial differential equations. An approach to possibly solving these equations, the " $\lambda$-method," is described in [Auckly and Kapitanski 2002, Auckly, Kapitanski, and White 2000]. Energy shaping for linear systems is considered in [Zenkov 2002].

\footnotetext{
*Professor, Department of Mathematics and Statistics, Queen's University, Kingston, ON K7L 3N6, CANADA

Email: andrew.lewis@queensu.ca, URL: http://www.mast.queensu.ca/ ªndrew/
} 
Additional contributions, in the setting of general rather than simple Lagrangians, have been made in the papers [Hamberg 1999, Hamberg 2000].

In this paper, we take an affine differential geometric approach to energy shaping. Of the work cited above, the approach we give here is closest in spirit to [Auckly, Kapitanski, and White 2000]. We see that what appears as a gyroscopic force in the method of controlled Lagrangians, and a change of interconnection in the IDA-PBC method, can also be thought of as a modification of the Levi-Civita affine connection to an affine connection that preserves energy in a sense that we make precise. Our allowing the closed-loop affine connection to possibly not be Levi-Civita captures the same sort of behaviour allowed in the IDA-PBC method by allowing the closed-loop Hamiltonian structure to be presymplectic rather than symplectic (i.e., the two-form may not be closed).

Our principal objective is to initiate a more systematic geometric exploration of the issues surrounding energy shaping. Page length restrictions make it impossible to report on all of the progress we have made here.

Notation. The identity map on a set $S$ is denoted $\operatorname{id}_{S}$. If $f: S \rightarrow T$ is a map of sets and if $A \subseteq S$, then $f \mid A$ denotes the restriction of $f$ to $A$.

The set of $(r, s)$-tensors on a $\mathbb{R}$-vector space $\mathrm{V}$ is denoted by $\mathrm{T}_{s}^{r}(\mathrm{~V})$. Given a $(0,2)$ tensor $A$ on $\mathrm{V}$, we define $A^{b}: \mathrm{V} \rightarrow \mathrm{V}^{*}$ by $\left\langle A^{b}(v) ; u\right\rangle=A(u, v)$ for $u, v \in \mathrm{V}$. If $A^{b}$ is an isomorphism, its inverse is denoted by $A^{\sharp}: \mathrm{V}^{*} \rightarrow \mathrm{V}$. The set of symmetric (resp. skewsymmetric) $(0,2)$-tensors on $\mathrm{V}$ is denoted by $\mathrm{TS}^{2}(\mathrm{~V})$ (resp. $\mathrm{T} \bigwedge^{2}(\mathrm{~V})$ ). We shall be dealing with multiple inner products, so it is necessary to reflect which inner product is being used to define notions such as symmetry of linear maps and orthogonality. We shall therefore write $\boldsymbol{M}$-symmetric or $\boldsymbol{M}$-orthogonal in such cases, where $M$ is an inner product. For $S \subseteq \mathrm{V}$ and $T \subseteq \mathrm{V}^{*}$, we denote

$$
\begin{aligned}
\operatorname{ann}(S) & =\left\{\alpha \in \mathrm{V}^{*} \mid \alpha(v)=0 \text { for all } v \in S\right\}, \\
\operatorname{coann}(T) & =\{v \in \mathrm{V} \mid \alpha(v)=0 \text { for all } \alpha \in T\} .
\end{aligned}
$$

All manifolds and maps will be assumed to be $C^{\infty}$, unless otherwise stated. The tangent bundle of a manifold $\mathrm{M}$ is denoted by $\pi_{\mathrm{TM}}: \mathrm{TM} \rightarrow \mathrm{M}$ and the cotangent bundle by $\pi_{\mathrm{T}^{*} \mathrm{Q}}: \mathrm{T}^{*} \mathrm{Q} \rightarrow \mathrm{Q}$. The zero vector in the tangent space at $x \in \mathrm{M}$ is denoted by $0_{x}$. The derivative of a map $f: \mathrm{M} \rightarrow \mathrm{N}$ between manifolds is denoted by $T f: \mathrm{TM} \rightarrow \mathrm{TN}$, and the restriction of $\mathrm{T} f$ to $\mathrm{T}_{x} \mathrm{M}$ is denoted by $T_{x} f$. The set of smooth functions on $\mathrm{M}$ is denoted by $C^{\infty}(\mathrm{M})$. The exterior derivative of a $k$-form $\alpha$ is denoted by $\boldsymbol{d} \alpha$. If $\pi: \mathrm{E} \rightarrow \mathrm{M}$ is a vector bundle, $\Gamma^{\infty}(\mathrm{E})$ denotes the set of $C^{\infty}$-sections of $\mathrm{E}$. The zero section of a vector bundle $\mathrm{E}$ is denoted by $Z(\mathrm{E})$. The vector bundle of vector bundle mappings over $\mathrm{id}_{\mathrm{M}}$ of a vector bundle $\pi: E \rightarrow M$ to itself are denoted by $\operatorname{End}(\mathrm{E})$.

\section{Problem formulation}

In this section, we define precisely the systems we study, and the problems we consider for these systems.

2.1. System definitions. Let us first define the control systems we are primarily interested in. 
2.1 Definition: A simple mechanical control system is a quadruple $(\mathrm{Q}, \mathrm{G}, V, \mathscr{F}=$ $\left.\left\{F^{1}, \ldots, F^{m}\right\}\right)$ where

(i) $\mathrm{Q}$ is an $n$-dimensional Hausdorff manifold,

(ii) $G$ is a Riemannian metric on $Q$,

(iii) $V$ is a function on $\mathrm{Q}$, and

(iv) $F^{1}, \ldots, F^{m}$ are one-forms on $\mathrm{Q}$, generating a subbundle of $\mathrm{T}^{*} \mathrm{Q}$ which we denote by $\mathcal{F}$.

The governing equations for a simple mechanical control system are

$$
\stackrel{\mathrm{G}}{\nabla}_{\gamma^{\prime}(t)} \gamma^{\prime}(t)=-\mathbb{G}^{\sharp} \circ \boldsymbol{d} V(\gamma(t))+\sum_{a=1}^{m} u_{a}(t) \mathbb{G}^{\sharp} \circ F^{a}(\gamma(t)),
$$

where $\stackrel{\mathrm{G}}{\nabla}$ is the Levi-Civita connection associated with G. It is possible that one might have additional external forces on the system. However, since this does not change the design problem (it does change the analysis problem for the closed-loop system), we do not complicate the problem by considering this.

Next we define the objective closed-loop system we seek. To discuss the character of the closed-loop systems we consider, we introduce some notation associated with a (0,3)-tensor field $A$ on $\mathrm{Q}$. We denote by $A^{\mathrm{b}}$ the fibre-quadratic map from TQ to $\mathrm{T}^{*} \mathrm{Q}$ given by

$$
\left\langle A^{\mathrm{b}}\left(v_{q}\right) ; u_{q}\right\rangle=A\left(u_{q}, v_{q}, v_{q}\right) .
$$

This notation is a natural adaptation of the usual flat map associated with a $(0,2)$-tensor field.

2.2 Definition: A forced simple mechanical system is a quadruple $(\mathrm{Q}, \mathbb{G}, V, F)$ where

(i) $\mathrm{Q}$ is an $n$-dimensional Hausdorff manifold,

(ii) $\mathbb{G}$ is a Riemannian metric on $Q$,

(iii) $V$ is a function on $Q$, and

(iv) $F: \mathrm{TQ} \rightarrow \mathrm{T}^{*} \mathrm{Q}$ is a bundle map over $\mathrm{id}_{\mathrm{Q}}$ called the external force.

An external force $F$ is quadratically gyroscopic if $F\left(v_{q}\right)=-B^{b}\left(v_{q}\right)$, where $B$ is a $(0,3)$-tensor field, called the quadratic gyroscopic tensor, satisfying $B\left(u_{q}, v_{q}, w_{q}\right)=$ $-B\left(v_{q}, u_{q}, w_{q}\right)$, for all $u_{q}, v_{q}, w_{q} \in \mathrm{TQ}$.

The equations of motion for a forced simple mechanical system are

$$
\stackrel{\mathrm{G}}{\nabla}_{\gamma^{\prime}(t)} \gamma^{\prime}(t)=-\mathbb{G}^{\sharp} \circ \boldsymbol{d} V(\gamma(t))+\mathbb{G}^{\sharp} \circ F\left(\gamma^{\prime}(t)\right),
$$

for a smooth curve $\gamma: I \rightarrow \mathrm{Q}$. In this paper, we shall not consider gyroscopic forces that are not quadratic, so we shall adopt the convention that a "quadratic gyroscopic" force is simply "gyroscopic." 
2.2. The energy shaping problem. We seek a closed-loop system that is a simple mechanical system with an external force that is a gyroscopic force. Thus the closed-loop system has the form $\left(\mathrm{Q}, \mathrm{G}_{\mathrm{cl}}, V_{\mathrm{cl}},-B_{\mathrm{cl}}^{\mathrm{b}}\right)$, where $B$ is a gyroscopic tensor. The reason for seeking this as a closed-loop system is that the stability of equilibria for such systems is well understood. The objective, therefore, is to design the control force $F\left(v_{q}\right)=\sum_{a=1}^{m} u_{a}\left(v_{q}\right) F^{a}(q)$ so that

$$
\begin{aligned}
& \stackrel{\mathrm{G}}{\nabla}_{\gamma^{\prime}(t)} \gamma^{\prime}(t)+\mathbb{G}^{\sharp} \circ \boldsymbol{d} V(\gamma(t))-\mathbb{G}^{\sharp} \circ F\left(\gamma^{\prime}(t)\right) \\
& =\stackrel{\mathrm{G}}{\mathrm{cl}}_{\gamma^{\prime}(t)} \gamma^{\prime}(t)+\mathbb{G}_{\mathrm{cl}}^{\sharp} \circ \boldsymbol{d} V_{\mathrm{cl}}(\gamma(t))+\mathbb{G}_{\mathrm{cl}}^{\sharp} \circ B_{\mathrm{cl}}^{b}\left(\gamma^{\prime}(t)\right),
\end{aligned}
$$

for any smooth curve $\gamma: I \rightarrow$ Q.

Let $\Lambda_{\mathrm{cl}}=\mathbb{G}^{b} \circ \mathbb{G}_{\mathrm{cl}}^{\sharp}$, noting that this is a vector bundle automorphism of $\mathrm{T}^{*} \mathrm{Q}$ over $\operatorname{id}_{\mathrm{Q}}$. The strategy we use to construct $F$ is as follows.

2.3 Definition: An energy shaping feedback for a simple mechanical control system $\Sigma=(\mathrm{Q}, \mathrm{G}, V, \mathscr{F})$ with closed-loop system $\Sigma_{\mathrm{cl}}=\left(\mathrm{Q}, \mathrm{G}_{\mathrm{cl}}, V_{\mathrm{cl}},-B_{\mathrm{cl}}^{b}\right)$ is given by $F: \mathrm{TQ} \rightarrow \mathcal{F}$ with $F=-F_{\text {kin }}-F_{\text {pot }}$, where

(i) $F_{\text {kin }}: \mathrm{TQ} \rightarrow \mathcal{F}$ has the property that

$$
\mathbb{G}^{\sharp} \circ F_{\text {kin }}\left(\gamma^{\prime}(t)\right)={\stackrel{\mathrm{G}_{\mathrm{cl}}}{\nabla}}_{\gamma^{\prime}(t)} \gamma^{\prime}(t)+\mathbb{G}_{\mathrm{cl}}^{\sharp} \circ B_{\mathrm{cl}}^{\mathrm{b}}\left(\gamma^{\prime}(t)\right)-\stackrel{\mathrm{G}}{\nabla}_{\gamma^{\prime}(t)} \gamma^{\prime}(t)
$$

for any smooth curve $\gamma$, and

(ii) $F_{\text {pot }}: \mathrm{Q} \rightarrow \mathcal{F}$ has the property that

$$
F_{\text {pot }}(\gamma(t))=\Lambda_{\mathrm{cl}} \circ \boldsymbol{d} V_{\mathrm{cl}}(\gamma(t))-\boldsymbol{d} V(\gamma(t))
$$

for any smooth curve $\gamma$.

2.4 Remarks: 1 . Note that we ask that the feedback be regarded as an $\mathcal{F}$-valued bundle map, rather than an $\mathbb{R}^{m}$-valued function on $T Q$. The latter certainly gives rise to the former. Indeed, given $u: T Q \rightarrow \mathbb{R}^{m}$, we define a $\mathcal{F}$-valued bundle map on TQ by

$$
F_{u}\left(v_{q}\right)=\sum_{a=1}^{m} u_{a}\left(v_{q}\right) F^{a}(q),
$$

where $F^{1}, \ldots, F^{m}$ are the one-forms comprising $\mathscr{F}$. The problem of inferring the existence of a $\mathbb{R}^{m}$-valued function on TQ given a $\mathcal{F}$-valued bundle map is not so straightforward. It is possible to do this in the case that $\mathcal{F}$ has constant rank, and is globally finitely generated. The latter condition is satisfied by our definition of a simple mechanical control system. However, the constant rank assumption will not generally hold, and this complicates the notion of feedback as we define it compared to feedback in the more usual sense.

2. Of course, one will generally include dissipative forces in the closed-loop system as well. We do not include this here, since our focus is principally on the shaping of the closedloop energy. However, there are some interesting issues to be considered for the addition of dissipative forces. 


\section{The geometry of potential energy shaping}

In this section, we describe feedback that shapes the potential energy. This seems to have first been considered in a general way in [van der Schaft 1986], although the geometric flavour of that work appears to have been lost in some of the recent literature on energy shaping.

We let $\Sigma=\left(\mathrm{Q}, \mathbb{G}, V, \mathscr{F}, \mathbb{R}^{m}\right)$ be a simple mechanical control system. While in practice one will typically design the closed-loop kinetic and potential energy together, for our purposes in this section it is convenient to suppose that the closed-loop kinetic energy $\mathbb{G}_{\mathrm{cl}}$ has already been defined. This will allow us to identify the important issues regarding potential shaping that must be considered when shaping the kinetic energy. Recall that the closed-loop kinetic energy metric gives rise to the bundle automorphism $\Lambda_{\mathrm{cl}}=\mathbb{G}^{b} \circ \mathbb{G}_{\mathrm{cl}}^{\sharp}$ of $T^{*} \mathrm{Q}$.

We need some notation concerning the nature of the one-forms defining the inputs for a simple mechanical control system. We suppose that $q_{0}$ is a regular point for $\mathcal{F}$. This implies that $q_{0}$ is also a regular point for $\mathcal{F}_{\mathrm{cl}} \triangleq \Lambda_{\mathrm{cl}}^{-1}(\mathcal{F})$. We denote by $\mathcal{F}_{\text {cl }}^{(\infty)}$ the largest integrable codistribution in $\mathcal{F}_{\mathrm{cl}}$. We suppose this codistribution to have constant rank near $q_{0}$, in which case we say that $\mathcal{F}$ is totally regular at $q_{0}$. We let $C^{\infty}(\mathrm{Q})_{\mathcal{F}_{\mathrm{cl}}}$ be the set of smooth functions $f$ on $Q$ having the property that $\boldsymbol{d} f \in \Gamma^{\infty}\left(\mathcal{F}_{\mathrm{cl}}\right)$. The following lemma follows essentially from Frobenius's Theorem (see [Bullo and Lewis 2004, Lemma 10.36]).

3.1 Lemma: If $\mathcal{F}$ is totally regular at $q_{0}$, then, for $f \in C^{\infty}(\mathrm{Q})$, the following statements are equivalent:

(i) there exists a neighbourhood $\mathcal{U}$ of $q_{0}$ for which $f \mid \mathcal{U} \in C^{\infty}(\mathcal{U})_{\mathcal{F}_{\mathrm{cl}} \mid}$;

(ii) there exists a neighbourhood $\mathcal{U}$ of $q_{0}$ for which $\boldsymbol{d}(f \mid \mathcal{U}) \in \Gamma^{\infty}\left(\mathcal{F}_{\mathrm{cl}}^{(\infty)} \mid \mathcal{U}\right)$.

Next we define

$$
\mathrm{PS}_{q_{0}}(\mathrm{Q})=\left\{V_{\mathrm{cl}} \oplus F_{\mathrm{cl}} \in C^{\infty}(\mathrm{Q}) \oplus \Gamma^{\infty}\left(\mathcal{F}_{\mathrm{cl}}\right) \mid \boldsymbol{d} V_{\mathrm{cl}}=F_{\mathrm{cl}}+\Lambda_{\mathrm{cl}}^{-1} \circ \boldsymbol{d} V, V_{\mathrm{cl}}\left(q_{0}\right)=0\right\} .
$$

Clearly, up to the normalisation of being zero at $q_{0}, \mathrm{PS}_{q_{0}}(\mathrm{Q})$ consists of solutions to the potential shaping problem, in the sense that, if $V_{\mathrm{cl}} \oplus F_{\mathrm{cl}} \in \mathrm{PS}_{q_{0}}(\mathrm{Q})$, then the closed-loop potential $V_{\mathrm{cl}}$ is achieved using the control force $F_{\mathrm{cl}}$. Next we define

$$
L\left(\mathrm{PS}_{q_{0}}(\mathrm{Q})\right)=\left\{f \oplus \alpha \in C^{\infty}(\mathrm{Q})_{\mathcal{F}_{\mathrm{cl}}} \oplus \Gamma^{\infty}\left(\mathcal{F}_{\mathrm{cl}}^{(\infty)}\right) \mid \boldsymbol{d} f=\alpha\right\}
$$

With this notation as backdrop, we state the following result, which tells us how many solutions the potential shaping problem has, and the form of those solutions, if it has one solution.

3.2 Proposition: If $(\mathrm{Q}, \mathrm{G}, V, \mathscr{F})$ is a simple mechanical control system, if $\mathrm{PS}_{q_{0}}(\mathrm{Q})$ is nonempty, and if $\mathcal{F}$ is totally regular at all points in $\mathrm{Q}$, then

(i) $\mathrm{PS}_{q_{0}}(\mathrm{Q})$ is an affine subspace of $C^{\infty}(\mathrm{Q}) \oplus \Gamma^{\infty}\left(\mathcal{F}_{\mathrm{cl}}\right)$ modelled on $L\left(\mathrm{PS}_{q_{0}}(\mathrm{Q})\right)$.

Furthermore, if the codistribution $\mathcal{F}_{\mathrm{cl}}$ is totally regular only at $q_{0}$, then

(ii) there exists a neighbourhood $\mathcal{U}$ of $q_{0}$ with the property that $L\left(\mathrm{PS}_{q_{0}}(\mathcal{U})\right)$ is naturally isomorphic to the closed sections of $\mathcal{F}_{\mathrm{cl}}^{(\infty)} \mid \mathrm{U}$. 
Proof: (i) Let $V_{\mathrm{cl}, 1} \oplus F_{\mathrm{cl}, 1}, V_{\mathrm{cl}, 2} \oplus F_{\mathrm{cl}, 2} \in \mathrm{PS}_{q_{0}}(\mathcal{U})$ and note that $\boldsymbol{d}\left(V_{\mathrm{cl}, 1}-V_{\mathrm{cl}, 2}\right)=F_{\mathrm{cl}, 1}-F_{\mathrm{cl}, 2}$. Thus

$$
V_{\mathrm{cl}, 1} \oplus F_{\mathrm{cl}, 1}-V_{\mathrm{cl}, 2} \oplus F_{\mathrm{cl}, 2} \in L\left(\mathrm{PS}_{q_{0}}(\mathcal{U})\right) .
$$

Also note that if $f \oplus \alpha \in L\left(\mathrm{PS}_{q_{0}}(\mathrm{Q})\right)$ and if $V_{\mathrm{cl}} \oplus F_{\mathrm{cl}} \in \mathrm{PS}_{q_{0}}(\mathrm{Q})$ then

$$
\boldsymbol{d}\left(V_{\mathrm{cl}}+f\right)=\left(F_{\mathrm{cl}}+\alpha\right)+\Lambda_{\mathrm{cl}}^{-1} \circ \boldsymbol{d} V,
$$

so that $\left(V_{\mathrm{cl}}+f\right) \oplus\left(F_{\mathrm{cl}}+\alpha\right) \in \mathrm{PS}_{q_{0}}(\mathcal{U})$. One can easily verify that these computations suffice for this part of the result.

(ii) We choose $\mathcal{U}$ to be connected and such that $\mathcal{F}$ is totally regular at each point in U. Consider the map from $L\left(\operatorname{PS}_{q_{0}}(\mathrm{Q})\right)$ to $\Gamma^{\infty}\left(\mathcal{F}_{\mathrm{cl}}^{(\infty)}\right)$ defined by $f \oplus \alpha \mapsto \alpha$. Given that $f\left(q_{0}\right)=0$ and that $\boldsymbol{d} f=\alpha$, it follows that this map is injective into the closed sections of $\mathcal{F}_{\text {cl }}^{(\infty)} \mid \mathcal{U}$. Regularity of $\mathcal{F}_{\text {cl }}^{(\infty)}$ on $\mathcal{U}$, and possibly shrinking $\mathcal{U}$ so that one can choose coordinates for which $\mathrm{d} q^{1}, \ldots, \mathrm{d} q^{k}$ are generators for $\mathcal{F}$, also establishes surjectivity of this projection onto the set of closed sections of $\mathcal{F}_{\mathrm{cl}}^{(\infty)} \mid \mathcal{U}$.

We shall not comment on this paper on conditions for $\mathrm{PS}_{q_{0}}(\mathrm{Q})$ to be nonempty, but refer to Section 5.1 for a formulation of this question as a geometric partial differential equation.

3.3 Remarks: Note that a general input codistribution $\mathcal{F}$ will have the property that $\mathcal{F}^{(\infty)}=$ $Z\left(\mathrm{~T}^{*} \mathrm{Q}\right)$. It does turn out that in many examples it actually holds that $\mathcal{F}^{(\infty)}=\mathcal{F}$, i.e., the input codistribution is integrable. Indeed, it is not uncommon to see this assumption made without comment. However, what Proposition 3.2 indicates is that, even in these cases, it might still be the case that $\mathcal{F}_{\mathrm{cl}}^{(\infty)}=Z\left(\mathrm{~T}^{*} \mathrm{Q}\right)$, and this severely limits the sort of potential shaping that can be achieved.

The special case where $\mathcal{F}$ is regular and codimension one has been singled out as being special in (at least) the papers [Auckly and Kapitanski 2002, Ortega, Spong, Gómez-Estern, and Blankenstein 2002]. The following simple result illustrates one reason why this case is of particular interest.

3.4 Lemma: Let $(\mathrm{Q}, \mathrm{G}, V, \mathscr{F})$ be a simple mechanical control system, suppose that $q_{0}$ is a regular point for $\mathcal{F}$, and suppose that $\operatorname{codim}\left(\mathcal{F}_{q_{0}}\right)=1$. Then there exists a neighbourhood $\mathcal{U}$ of $q_{0}$ such that

(i) $\mathcal{F}^{(\infty)}|\mathcal{U}=\mathcal{F}| \mathcal{U}$ and

(ii) $\mathcal{F}_{\mathrm{cl}}^{(\infty)}\left|\mathcal{U}=\mathcal{F}_{\mathrm{cl}}\right| \mathcal{U}$.

Proof: This follows simply because if $q_{0}$ is a regular point for a codimension one codistribution, then the codistribution is integrable in a neighbourhood of $q_{0}$ by Frobenius's Theorem.

3.5 Remark: Many of the examples worked out in the literature have had the property that $\operatorname{dim}(\mathrm{Q})=2$ and $\operatorname{rank}(\mathcal{F})=1$. The preceding lemma illustrates why, in these examples, the authors were able to disregard the issues raised in Remark 3.3. 


\section{An affine connection formulation of kinetic energy shaping}

In this section we consider the shaping of the closed-loop kinetic energy metric. We recall from Definition 2.3 that the objective is to find a Riemannian metric $\mathbb{G}_{\mathrm{cl}}$ and a gyroscopic force $B_{\mathrm{cl}}$ for which

$$
\stackrel{\mathrm{G}}{\mathrm{cl}}_{\gamma^{\prime}(t)} \gamma^{\prime}(t)+\mathbb{G}_{\mathrm{cl}}^{\sharp} \circ B_{\mathrm{cl}}^{b}\left(\gamma^{\prime}(t)\right)-\stackrel{\mathrm{G}}{\nabla}_{\gamma^{\prime}(t)} \gamma^{\prime}(t) \in \mathbb{G}^{\sharp}(\mathcal{F})_{\gamma(t)}
$$

for any smooth curve $\gamma$. The point of view we take here is that we design a closed-loop affine connection with certain properties relative to the desired closed-loop kinetic energy. In order to do this, it is convenient to first say some things about $(0,3)$-tensor fields.

4.1. Decompositions of $(\mathbf{0}, \mathbf{3})$-tensors. We let $(Q, G)$ be a Riemannian manifold. Note that, if $\nabla$ is any other affine connection on $Q$, then we have

$$
\mathrm{G}\left(\nabla_{X} Y, Z\right)=\mathbb{G}\left(\stackrel{\mathrm{G}}{\nabla}_{X} Y, Z\right)+D_{\nabla, \mathrm{G}}(Z, X, Y)
$$

for some uniquely defined $(0,3)$-tensor field $D_{\nabla, \mathbb{G}}$. If we decompose $D_{\nabla, \mathbb{G}}$ into its symmetric and skew-symmetric parts with respect to the last two entries, we have

$$
\begin{aligned}
D_{\nabla, \mathrm{G}}(X, Y, Z)=\underbrace{\frac{1}{2}\left(D_{\nabla, \mathrm{G}}(X, Y, Z)+D_{\nabla, \mathrm{G}}(X, Z, Y)\right)}_{S_{\nabla, \mathrm{G}}(X, Y, Z)} \\
+\underbrace{\frac{1}{2}\left(D_{\nabla, \mathrm{G}}(X, Y, Z)-D_{\nabla, \mathrm{G}}(X, Z, Y)\right)}_{T_{\nabla, \mathrm{G}}(X, Y, Z)},
\end{aligned}
$$

so defining two $(0,3)$-tensor fields $S_{\nabla, \mathbb{G}}$ and $T_{\nabla, \mathbb{G}}$. Note that, if $T$ is the torsion tensor for $\nabla$, then we have

$$
2 T_{\nabla, \mathrm{G}}(X, Y, Z)=\mathbb{G}(Z, T(X, Y)) .
$$

Also note that the geodesics of $\nabla$ depend only on $S_{\nabla, \mathrm{G}}$ and not on $T_{\nabla, \mathrm{G}}$. More precisely, geodesics $\gamma$ of $\nabla$ satisfy

$$
\nabla_{\gamma^{\prime}(t)} \gamma^{\prime}(t)=\stackrel{\mathrm{G}}{\nabla}_{\gamma^{\prime}(t)} \gamma^{\prime}(t)+\mathbb{G}^{\sharp} \circ S_{\nabla, \mathrm{G}}^{\mathrm{b}}\left(\gamma^{\prime}(t)\right) .
$$

The preceding discussion motivates the following definitions.

4.1 Definition: A $(0,3)$-tensor field $A$ on $\mathrm{Q}$ is

(i) gyroscopic if $A(X, Y, Z)=-A(Y, X, Z)$ for all $X, Y, Z \in \Gamma^{\infty}(\mathrm{TQ})$ (this is a repetition of our former definition of a gyroscopic tensor field), is

(ii) geodesic if if $A(X, Y, Z)=A(X, Z, Y)$ for all $X, Y, Z \in \Gamma^{\infty}(\mathrm{TQ})$, is

(iii) torsional if $A(X, Y, Z)=-A(X, Z, Y)$ for all $X, Y, Z \in \Gamma^{\infty}(\mathrm{TQ})$, and is

(iv) skew if $A \in \Gamma^{\infty}\left(\mathrm{T} \bigwedge^{3}(\mathrm{TQ})\right)$.

The discussion preceding the definition can now be summarised as follows. 
4.2 Lemma: If $(\mathrm{Q}, \mathrm{G})$ is a Riemannian manifold and if $\nabla$ is an affine connection on $\mathrm{Q}$, then there exists a unique geodesic tensor field $S_{\nabla, \mathrm{G}}$ and a unique torsional tensor field $T_{\nabla, \mathrm{G}}$ satisfying $\mathrm{G}\left(\nabla_{X} Y, Z\right)=\mathrm{G}\left(\nabla_{X}^{\mathrm{G}} Y, Z\right)+S_{\nabla, \mathrm{G}}(Z, X, Y)+T_{\nabla, \mathrm{G}}(Z, X, Y)$, for $X, Y, Z \in$ $\Gamma^{\infty}(\mathrm{TQ})$.

Now we wish to decompose an arbitrary $(0,3)$-tensor in a particular way. It is convenient now to simply work with a $\mathbb{R}$-vector space $\mathrm{V}$, adapting in the obvious way the definitions for gyroscopic, geodesic, torsional, and skew tensors. Let us denote by $\operatorname{Gyr}(\mathrm{V})$ and $\operatorname{Tor}(\mathrm{V})$ the set of gyroscopic and torsional tensors on $\mathrm{V}$. We shall also require the symmetrising and skew-symmetrising maps. Thus, for $A \in \mathrm{T}_{k}^{0}(\mathrm{~V})$, we define

$$
\operatorname{Sym}(A)\left(v_{1}, \ldots, v_{k}\right)=\frac{1}{k !} \sum_{\sigma \in S_{k}} A\left(v_{\sigma(1)}, \ldots, v_{\sigma(k)}\right)
$$

and

$$
\operatorname{Alt}(A)\left(v_{1}, \ldots, v_{k}\right)=\frac{1}{k !} \sum_{\sigma \in S_{k}}(-1)^{\operatorname{sgn}(\sigma)} A\left(v_{\sigma(1)}, \ldots, v_{\sigma(k)}\right) .
$$

Here $S_{k}$ is the permutation group on $k$ symbols, and $\operatorname{sgn}(\sigma)$ is the parity of the permutation $\sigma$.

The following decomposition result will be useful. There are natural extensions of this result to higher-degree tensors, but we shall only need what we present here.

4.3 Lemma: For a finite-dimensional $\mathbb{R}$-vector space $\mathrm{V}$, the following statements hold:

(i) $\mathrm{T}_{3}^{0}(\mathrm{~V})=\mathrm{TS}^{3}(\mathrm{~V}) \oplus \mathrm{T} \bigwedge^{3}(\mathrm{~V}) \oplus(\operatorname{Gyr}(\mathrm{V}) \cap \operatorname{ker}(\mathrm{Alt})) \oplus(\operatorname{Tor}(\mathrm{V}) \cap \operatorname{ker}(\mathrm{Alt}))$;

(ii) in particular, $\operatorname{ker}(\operatorname{Sym})=\mathrm{T} \bigwedge^{3}(\mathrm{~V}) \oplus(\operatorname{Gyr}(\mathrm{V}) \cap \operatorname{ker}(\mathrm{Alt})) \oplus(\operatorname{Tor}(\mathrm{V}) \cap \operatorname{ker}($ Alt $))$;

(iii) for $A \in \operatorname{ker}(\mathrm{Sym})$, the decomposition $A=S_{A}+\Omega_{A}+B_{A}+T_{A}, S_{A} \in \mathrm{TS}^{3}(\mathrm{~V})$, $\Omega_{A} \in \mathrm{T} \bigwedge^{3}(\mathrm{~V}), B_{A} \in \operatorname{Gyr}(\mathrm{V}) \cap \operatorname{ker}(\mathrm{Alt}), T_{A} \in \operatorname{Tor}(\mathrm{V} \cap \operatorname{ker}($ Alt $)$, is given explicitly by

$$
\begin{aligned}
& S_{A}(u, v, w)=\frac{1}{6}(A(u, v, w)+A(u, w, v)+A(v, w, u) \\
& \quad+A(v, u, w)+A(w, u, v)+A(w, v, u)) \\
& \Omega_{A}(u, v, w)=\frac{1}{6}(A(u, v, w)-A(u, w, v)+A(v, w, u) \\
& \quad-A(v, u, w)+A(w, u, v)-A(w, v, u)) \\
& B_{A}(u, v, w)=\frac{1}{3}(A(u, v, w)+A(u, w, v) \\
& \quad-A(v, u, w)-A(v, w, u)) \\
& T_{A}(u, v, w)=\frac{1}{3}(A(u, v, w)+A(v, u, w) \\
& \quad-A(u, w, v)-A(w, u, v)) .
\end{aligned}
$$

Proof: Note that Sym: $\mathrm{T}_{3}^{0}(\mathrm{~V}) \rightarrow \mathrm{TS}^{3}(\mathrm{~V})$ is a projection, so that we have $\mathrm{T}_{3}^{0}(\mathrm{~V})=$ $\mathrm{TS}^{3}(\mathrm{~V}) \oplus \operatorname{ker}(\mathrm{Sym})$. Thus part (i) will follow if part (ii) is proved. It is readily checked that $\mathrm{T} \bigwedge^{3}(\mathrm{~V}), \operatorname{Gyr}(\mathrm{V}), \operatorname{Tor}(\mathrm{V}) \subseteq \operatorname{ker}(\operatorname{Sym})$. Furthermore, the explicit expressions for $\Omega_{A}$, $B_{A}$, and $T_{A}$ are verified by direct computation to satisfy $A=\Omega_{A}+B_{A}+T_{A}$, provided that $A \in \operatorname{ker}(\mathrm{Sym})$. Since $\Omega$ is torsional (and gyroscopic), it follows that $\operatorname{ker}(\mathrm{Sym})=$ $\operatorname{Gyr}(\mathrm{V})+\operatorname{Tor}(\mathrm{V})$. Next note that Alt: $\operatorname{ker}(\mathrm{Sym}) \rightarrow \mathrm{T} \bigwedge^{3}(\mathrm{~V})$ is a projection, so that $\operatorname{ker}(\operatorname{Sym})=\mathrm{T} \bigwedge^{3}(\mathrm{~V}) \oplus(\operatorname{ker}($ Alt $) \cap \operatorname{ker}(\operatorname{Sym}))$. It therefore follows that

$$
\operatorname{ker}(\operatorname{Sym})=\mathrm{T} \bigwedge^{3}(\mathrm{~V}) \oplus((\operatorname{Gyr}(\mathrm{V})+\operatorname{Tor}(\mathrm{V})) \cap \operatorname{ker}(\mathrm{Alt}))
$$


Next, suppose that $A \in \operatorname{Gyr}(\mathrm{V}) \cap \operatorname{Tor}(\mathrm{V}) \cap \operatorname{ker}($ Alt). Since $A$ is gyroscopic and torsional, we have

$$
A(u, v, w)=-A(v, u, w)=A(w, u, v)=-A(w, v, u),
$$

so showing that $A$ is skew-symmetric in all entries i.e., that $A \in \mathrm{T} \bigwedge^{3}(\mathrm{~V})$. Since $A \in \operatorname{ker}(\mathrm{Alt})$, we have $A=0$. Part (ii), and therefore part (i), now follows.

The proof of part (iii) is merely a matter of directly checking that $S_{A}, \Omega_{A}, B_{A}$, and $T_{A}$ have the stated properties.

The following (now) simple result will also be helpful in shedding some light on the methodology of this section.

4.4 Corollary: If $\mathrm{V}$ is a finite-dimensional $\mathbb{R}$-vector space and if $A \in \operatorname{Gyr}(\mathrm{V})$, then there is a unique decomposition $B_{A}=G_{A}+\Omega_{A}$ where $G_{A}$ is gyroscopic and geodesic and $\Omega_{A}$ is skew.

Proof: Every (0,3)-tensor may be uniquely decomposed as $A=G_{A}+T_{A}$ where $G_{A}$ is geodesic and $T_{A}$ is torsional. Explicitly

$$
\begin{aligned}
G_{A}(X, Y, Z) & =\frac{1}{2}(A(X, Y, Z)+A(X, Z, Y)), \\
T_{A}(X, Y, Z) & =\frac{1}{2}(A(X, Y, Z)-A(X, Z, Y)) .
\end{aligned}
$$

Since $A \in \operatorname{Gyr}(\mathrm{V}) \subseteq \operatorname{ker}(\operatorname{Sym})$, by Lemma 4.3 we have $A=B_{A}+T_{A}$ where $B_{A} \in \operatorname{Gyr}(\mathrm{V}) \cap$ $\operatorname{ker}(\mathrm{Alt})$ and $T_{A}$ is torsional. From the uniqueness of the torsional part of the decomposition $A=G_{A}+T_{A}$, we conclude that $B_{A}=G_{A}$. Thus $G_{A}$ is gyroscopic and geodesic. It follows that $T_{A}$ is gyroscopic and torsional, and therefore skew.

4.2. Energy preserving affine connections. In this section, we extend the definitions of Alt and Sym to being defined as vectors bundle maps. We also extend the previous notation for gyroscopic and torsional tensors to tensor bundles by $\operatorname{Gyr}(\mathrm{TQ})$ and $\operatorname{Tor}(\mathrm{TQ})$. Thus these are both now subbundles of $\mathrm{T}_{3}^{0}(\mathrm{TQ})$.

Let $G$ be a Riemannian metric on $Q$. Define the kinetic energy associated to $\mathbb{G}$ by $\mathrm{KE}_{\mathbb{G}}\left(v_{q}\right)=\frac{1}{2} \mathbb{G}\left(v_{q}, v_{q}\right)$. An affine connection $\nabla$ on $\mathrm{Q}$ is $\mathbf{G}$-energy preserving if, for every geodesic $\gamma: I \rightarrow \mathrm{Q}$ for $\nabla$, it holds that $\mathscr{L}_{\gamma^{\prime \prime}(t)}\left(\mathrm{KE}_{\mathrm{G}}\left(\gamma^{\prime}(t)\right)\right)=0$.

The following result now characterises energy preserving affine connections, recalling the definition of $D_{\nabla, \mathrm{G}}$ from (4.2), and extending the definition of Sym from tensors to tensor fields in the obvious way.

4.5 Proposition: For a Riemannian manifold $(\mathrm{Q}, \mathrm{G})$ and an affine connection $\nabla$ on $\mathrm{Q}$, the following statements are equivalent:

(i) $\nabla$ is G-energy preserving;

(ii) $\nabla \mathbb{G} \in \Gamma^{\infty}(\operatorname{ker}(\mathrm{Sym}))$;

(iii) $D_{\nabla, \mathrm{G}} \in \Gamma^{\infty}(\operatorname{ker}(\mathrm{Sym}))$.

(iv) there exists tensor fields $\Omega_{\nabla, \mathrm{G}} \in \Gamma^{\infty}\left(\mathrm{T} \bigwedge^{3}(\mathrm{TQ})\right), B_{\nabla, \mathrm{G}} \in \Gamma^{\infty}((\mathrm{Gyr}(\mathrm{TQ}) \cap \operatorname{ker}(\mathrm{Alt})))$, and $\hat{T}_{\nabla, \mathrm{G}} \in \Gamma^{\infty}((\operatorname{Tor}(\mathrm{TQ}) \cap \operatorname{ker}(\mathrm{Alt})))$ such that

$$
\mathrm{G}\left(\nabla_{X} Y, Z\right)=\mathbb{G}\left(\nabla_{X}^{\mathrm{G}} Y, Z\right)+B_{\nabla, \mathrm{G}}(Z, X, Y)+\hat{T}_{\nabla, \mathrm{G}}(Z, X, Y)+\Omega_{\nabla, \mathrm{G}}(Z, X, Y),
$$

for all $X, Y, Z \in \Gamma^{\infty}(\mathrm{TQ})$. 
Proof: (i) $\Longrightarrow$ (ii) Let $v_{q} \in \mathrm{T}_{q} \mathrm{Q}$ and let $\gamma$ be a geodesic satisfying $\gamma^{\prime}(0)=v_{q}$. We then have

$$
\begin{aligned}
\mathscr{L}_{\gamma^{\prime \prime}(t)}\left(\mathrm{KE}_{\mathbb{G}}\left(\gamma^{\prime}(t)\right)\right)= & \frac{1}{2} \nabla_{\gamma^{\prime}(t)} \mathbb{G}\left(\gamma^{\prime}(t), \mathbb{G}^{\prime}(t)\right) \\
& +\mathbb{G}\left(\nabla_{\gamma^{\prime}(t)} \gamma^{\prime}(t), \gamma^{\prime}(t)\right) \\
= & \frac{1}{2} \nabla_{\gamma^{\prime}(t)} \mathbb{G}\left(\gamma^{\prime}(t), \mathbb{G}^{\prime}(t)\right) .
\end{aligned}
$$

Evaluating at $t=0$ gives $\nabla \mathrm{G}\left(v_{q}, v_{q}, v_{q}\right)=0$. By polarisation, the equalities

$$
\begin{aligned}
& \nabla \mathrm{G}\left(v_{q}, v_{q}, v_{q}\right)=0, \text { for all } v_{q} \in \mathrm{T}_{q} \mathrm{Q}, \\
& \operatorname{Sym}(\nabla \mathbb{G})(q)=0
\end{aligned}
$$

are equivalent. Therefore, (ii) follows.

(ii) $\Longrightarrow$ (iii) A straightforward calculation (see [Lewis 1998, Lemma 6.10]) gives

$$
\begin{aligned}
(\nabla \mathbb{G})(X, Y, Z) & =\left(\nabla_{X} \mathbb{G}\right)(Y, Z) \\
& =-D_{\nabla, \mathrm{G}}(Z, X, Y)-D_{\nabla, \mathrm{G}}(Y, X, Z) .
\end{aligned}
$$

A direct calculation using the definition of $\operatorname{Sym}(\nabla \mathbb{G})$ yields

$$
\operatorname{Sym}(\nabla \mathbb{G})(X, Y, Z)=-\frac{1}{3} \operatorname{Sym}\left(D_{\nabla, \mathbb{G}}\right)(X, Y, Z)
$$

for $X, Y, Z \in \Gamma^{\infty}(\mathrm{TQ})$. This gives this part of the proof.

(iii) $\Longrightarrow$ (iv) This follows directly from Lemma 4.3 , defining $B_{\nabla, G}$ by

$$
\begin{aligned}
B_{\nabla, \mathrm{G}}(X, Y, Z)=\frac{1}{3}\left(D_{\nabla, \mathrm{G}}(X, Y, Z)+D_{\nabla, \mathrm{G}}(X, Z, Y)\right. & \\
& \left.-D_{\nabla, \mathrm{G}}(Y, X, Z)-D_{\nabla, \mathrm{G}}(Y, Z, X)\right),
\end{aligned}
$$

defining $\hat{T}_{\nabla, \mathrm{G}}$ by

$$
\begin{aligned}
\hat{T}_{\nabla, \mathrm{G}}(X, Y, Z)=\frac{1}{3}\left(D_{\nabla, \mathrm{G}}(X, Y, Z)+D_{\nabla, \mathrm{G}}(Y, X, Z)\right) & \\
& \left.-D_{\nabla, \mathrm{G}}(X, Z, Y)-D_{\nabla, \mathrm{G}}(Z, X, Y)\right),
\end{aligned}
$$

and defining $\Omega_{\nabla, G}$ by

$$
\begin{aligned}
\Omega_{\nabla, \mathrm{G}}(X, Y, Z)=\frac{1}{6}\left(D_{\nabla, \mathrm{G}}(\right. & X, Y, Z)-D_{\nabla, \mathrm{G}}(X, Z, Y) \\
& +D_{\nabla, \mathrm{G}}(Y, Z, X)-D_{\nabla, \mathrm{G}}(Y, X, Z) \\
& +D_{\nabla, \mathrm{G}}(Z, X, Y)-D_{\nabla, \mathrm{G}}(Z, Y, X) .
\end{aligned}
$$

(iv) $\Longrightarrow$ (i) Since $\hat{T}_{\nabla, G}$ and $\Omega_{\nabla, G}$ are torsional, the geodesics $\gamma$ for $\nabla$ satisfy the equation

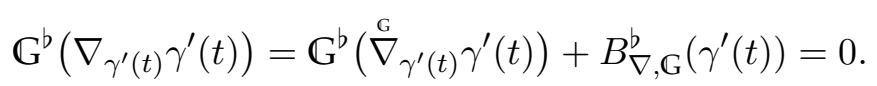

Therefore, for a solution $\gamma$ to this equation, we have

$$
\begin{aligned}
\mathscr{L}_{\gamma^{\prime \prime}(t)}\left(\mathrm{KE}_{\mathbb{G}}\left(\gamma^{\prime}(t)\right)=\right. & \frac{1}{2}\left({\stackrel{\mathrm{G}}{\gamma^{\prime}(t)}} \mathrm{G}\right)\left(\gamma^{\prime}(t), \gamma^{\prime}(t)\right) \\
& +\mathrm{G}\left(\nabla_{\gamma^{\prime}(t)} \gamma^{\prime}(t), \gamma^{\prime}(t)\right) \\
= & -B_{\nabla, \mathrm{G}}\left(\gamma^{\prime}(t), \gamma^{\prime}(t), \gamma^{\prime}(t)\right)=0,
\end{aligned}
$$

using the fact that $B_{\nabla, \mathrm{G}}$ is skew-symmetric in the first two arguments. Thus $\nabla$ is $\mathrm{G}$-energy preserving. 
It is useful to pull from the proof the exact relationship between the concepts of an energy preserving connection and of a gyroscopic tensor field. Note that a gyroscopic tensor determines the corresponding energy preserving affine connection only up to torsion.

4.6 Corollary: If $(\mathrm{Q}, \mathrm{G})$ is a Riemannian manifold, then the following statements hold:

(i) if $\nabla$ is a G-energy preserving affine connection, then the gyroscopic tensor $B_{\nabla, \mathrm{G}}$ and the torsional tensor $T_{\nabla, \mathrm{G}}$ defined by (4.5) and (4.6) are the unique such tensor fields satisfying the conditions specified in part (iv) of Proposition 4.5;

(ii) if $B$ is a gyroscopic tensor field, then there exists a unique torsion-free G-energy preserving affine connection $\nabla$ satisfying

$$
\mathbb{G}\left(\nabla_{X} X, Y\right)=\mathbb{G}\left(\stackrel{\mathrm{G}}{\nabla}_{X} X, Y\right)+B(Y, X, X),
$$

$X, Y \in \Gamma^{\infty}(\mathrm{TQ})$;

(iii) with $B$ and $\nabla$ as in part (ii), we have

$$
\mathrm{G}\left(\nabla_{X} Y, Z\right)=\mathbb{G}\left(\nabla_{X}^{\mathrm{G}} Y, Z\right)+B_{0}(Z, X, Y),
$$

$X, Y, Z \in \Gamma^{\infty}(\mathrm{TQ})$, where $B_{0}=B-\operatorname{Alt}(B)$ is gyroscopic and geodesic.

Proof: Only (ii) is potentially not obvious. To prove this part of the result, let $B_{\nabla, \mathrm{G}}=$ $B-\operatorname{Alt}(B)$ be the projection of $B$ onto $\operatorname{ker}\left(\right.$ Alt), so that $B=B_{\nabla, \mathrm{G}} \oplus \operatorname{Alt}(B)$ is the decomposition of $B$ as a section of $\mathrm{T}^{3}(\mathrm{TQ}) \oplus(\operatorname{Gyr}(\mathrm{TQ}) \cap \operatorname{ker}($ Alt $))$. Then we define $\nabla$ by

$$
\mathrm{G}\left(\nabla_{X} Y, Z\right)=\mathbb{G}\left(\nabla_{X}^{\mathrm{G}} Y, Z\right)+B_{\nabla, \mathrm{G}}(Z, X, Y) .
$$

Note that our use of the notation $B_{\nabla, \mathbb{G}}$ is consistent with that in Proposition 4.5. Also note that Lemmas 4.2 and 4.3 imply that $\nabla$ is torsion free. Furthermore, $\operatorname{since} \operatorname{Alt}(B)$ is torsional,

$$
\mathbb{G}\left(\nabla_{X} Y, Z\right)=\mathbb{G}\left(\nabla_{X}^{\mathrm{G}} Y, Z\right)+B(Z, X, Y) .
$$

Any other affine connection satisfying this relationship can only differ from $\nabla$ by torsion, giving the uniqueness asserted.

4.3. A reformulation of the kinetic energy shaping problem. The above results allow the following reformulation of the kinetic energy part of the energy shaping problem.

4.7 Problem: Given a Riemannian manifold (Q, G), find

(i) a Riemannian metric $\mathbb{G}_{\mathrm{cl}}$ and

(ii) a $\mathrm{G}_{\mathrm{cl}}$-energy preserving affine connection $\stackrel{\mathrm{cl}}{\nabla}$

with the property that $\stackrel{\text { cl }}{\nabla}_{\gamma^{\prime}(t)} \gamma^{\prime}(t)-\stackrel{\mathrm{G}}{\nabla}_{\gamma^{\prime}(t)} \gamma^{\prime}(t) \in \mathbb{G}^{\sharp}(\mathcal{F})_{\gamma(t)}$. for any smooth curve $\gamma$

In papers on energy shaping, it appears to be de rigeur to ensure that one's approach is at least as general as others'. Here we briefly indicate the relationship between our approach and that of the method of controlled Lagrangians, and the IDA-PBC method. Indeed, we merely state the following result, whose proof is rather evident, given some familiarity with the papers of [Blankenstein, Ortega, and van der Schaft 2002, Chang, Bloch, Leonard, Marsden, and Woolsey 2002]. 
4.8 Proposition: Problem 4.7 is equivalent to the kinetic energy shaping of the method of controlled Lagrangians and the IDA-PBC method, provided that

(i) in the method of controlled Lagrangians one restricts consideration to gyroscopic forces as we define them, and that

(ii) in the IDA-PBC method, one restricts the closed-loop symplectic structure $\Omega_{\mathrm{cl}}$ to be of the form

$$
\Omega_{\mathrm{cl}, \alpha_{q}}(X, Y)=\Omega_{0}(X, Y)
$$

$$
+B\left(T \pi_{\mathrm{T}^{*} \mathrm{Q}}(X), T \pi_{\mathrm{T}^{*} \mathrm{Q}}(Y), \Lambda_{\mathrm{cl}}^{*}\left(\alpha_{q}\right)\right),
$$

where $\alpha_{q} \in \mathrm{T}_{q}^{*} \mathrm{Q}, X, Y \in \mathrm{T}_{\alpha_{q}} \mathrm{~T}^{*} \mathrm{Q}$, where $\Omega_{0}$ is the canonical cotangent bundle symplectic structure, where $B$ is a gyroscopic tensor on $\mathrm{Q}$, and where $\pi_{\mathrm{T} * \mathrm{Q}}$ is the cotangent bundle projection.

To the best of our knowledge, there have appeared no examples in the literature, using the method of controlled Lagrangians or the IDA-PBC method, and which do not fit into the affine connection framework for kinetic energy shaping that we have formulated.

\section{Partial differential equation formulations}

In this section it will be convenient to think of Riemannian metrics as independent variables. For this reason, we shall write the Riemannian metric for the open-loop system as $\mathbb{G}_{\mathrm{ol}}$, reserving the symbol $\mathbb{G}$ for a generic Riemannian metric. In like manner, $V$ will denote a generic potential function, with $V_{\mathrm{ol}}$ standing for the open-loop potential.

The determination of a closed-loop Riemannian metric $\mathbb{G}_{\mathrm{cl}}$ and a closed-loop potential $V_{\mathrm{cl}}$ is difficult, since the conditions restricting the available closed-loop data come in the form of nonlinear partial differential equations. One way to study the integrability of these equations is to use the methodology initiated by Spencer [1962a] and Spencer [1962b], developed further by many authors, including Goldschmidt [1967], and reported on in the monograph [Bryant, Chern, Gardner, Goldschmidt, and Griffiths 1991]. While this method is nontrivial to apply, it has proven useful in getting insights into exactly the sorts of geometric problems such as are posed for energy shaping. Therefore, in this section we put the energy shaping problem into a framework where this theory can be applied, assuming the reader to be familiar with the treatment in [Bryant, Chern, Gardner, Goldschmidt, and Griffiths 1991]. Subsequent work will be directed to gaining insights into the sorts of closed-loop energies that are available using energy shaping.

5.1. The partial differential equation of potential energy shaping. In Section 3 we addressed the question of the number of solutions to the potential shaping problem, once one has performed kinetic energy shaping. In the section, we give a geometric formulation of the partial differential equation describing the existence of a solution to the potential shaping problem. We recall the definition $\Lambda_{\mathrm{cl}}=\mathbb{G}^{b} \circ \mathbb{G}_{\mathrm{cl}}^{\sharp}$.

We adopt the point of view that a $k$ th-order partial differential equation is a fibred submanifold of the $k$-jet bundle of a fibre bundle. We need, therefore, to indicate what 
the fibre bundle is for our problem, and what the submanifold is of the jet bundle. The space of variables for the problem we take to be the vector bundle $\mathcal{F}_{\mathrm{cl}}=\Lambda_{\mathrm{cl}}(\mathcal{F})$, which we assume to have constant rank. The exact relationship between this space of variables and the set of achievable closed-loop potentials is given in Proposition 5.1 below. We let $\mathrm{J}^{1}\left(\mathcal{F}_{\mathrm{cl}}\right)$ denote the bundle of 1 -jets of sections of $\mathcal{F}_{\mathrm{cl}}$. A point in $\mathrm{J}^{1}\left(\mathcal{F}_{\mathrm{cl}}\right)$ we shall denote by $j^{1} F_{\mathrm{cl}}(q)$. We note that the exterior derivative of an $\mathcal{F}_{\mathrm{cl}}$-valued one-form can be thought of as a mapping from $\mathrm{J}^{1}\left(\mathcal{F}_{\mathrm{cl}}\right)$ to $\mathrm{T} \bigwedge^{2}(\mathrm{TQ})$. Explicitly, in coordinates, this mapping is given by $\left(q^{i}, F_{\mathrm{cl}, j}, F_{\mathrm{cl}, k, \ell}\right) \mapsto\left(q^{i}, F_{\mathrm{cl}, j, k}-F_{\mathrm{cl}, k, j}\right)$, where $F_{\mathrm{cl}, k, \ell}$ means the partial derivative of $F_{\mathrm{cl}, k}$ with respect to $q^{\ell}$, thinking of this as a coordinate for $\mathrm{J}^{1}\left(\mathcal{F}_{\mathrm{cl}}\right)$. This map from $\mathrm{J}^{1}\left(\mathcal{F}_{\mathrm{cl}}\right)$ to $\mathrm{T} \bigwedge^{2}(\mathrm{TQ})$ we denote by $\boldsymbol{d}_{1}$. We then define a fibred submanifold of $\mathrm{J}^{1}\left(\mathcal{F}_{\mathrm{cl}}\right)$ by

$$
P_{\mathrm{PS}}\left(V_{\mathrm{ol}}\right)_{q}=\left\{j^{1} F_{\mathrm{cl}}(q) \mid \boldsymbol{d}_{1}\left(j^{1} F_{\mathrm{cl}}(q)\right)=-\boldsymbol{d}\left(\Lambda_{\mathrm{cl}} \circ \boldsymbol{d} V_{\mathrm{ol}}\right)(q)\right\} .
$$

The following result gives the formulation of the potential shaping problem as a geometric partial differential equation.

5.1 Proposition: Suppose that the first cohomology group of $\mathrm{Q}$ is zero. Then a function $V_{\mathrm{cl}}$ is a possible closed-loop potential function if and only if $\boldsymbol{d} V_{\mathrm{cl}}=F_{\mathrm{cl}}+\Lambda_{\mathrm{cl}} \circ \boldsymbol{d} V_{\mathrm{ol}}$ where $F_{\mathrm{cl}}$ is a section of $\mathcal{F}_{\mathrm{cl}}$ having the property that $j^{1} F_{\mathrm{cl}}$ takes values in $P_{\mathrm{PS}}\left(V_{\mathrm{ol}}\right)$.

Proof: A closed-loop potential $V_{\mathrm{cl}}$ has the property that there is a section $F$ of $\mathcal{F}$ satisfying $F(q)=\Lambda_{\mathrm{cl}}{ }^{\circ} \boldsymbol{d} V_{\mathrm{cl}}(q)-\boldsymbol{d} V_{\mathrm{ol}}(q)$. Since the first cohomology group of $\mathrm{Q}$ is zero, this is equivalent to asserting the existence of a section $F_{\mathrm{cl}}$ of $\mathcal{F}_{\mathrm{cl}}$ having the property that $F_{\mathrm{cl}}+\Lambda_{\mathrm{cl}}^{-1} \circ \boldsymbol{d} V_{\mathrm{ol}}$ is closed. This, however, exactly describes what it means for the 1-jet of $F_{\mathrm{cl}}$ to take values in $P_{\mathrm{PS}}\left(V_{\mathrm{ol}}\right)$.

5.2. Affine connections as sections of a bundle. In this section, we come to an understanding of what is meant by an affine connection, but in a very particular way. Namely, we realise an affine connection as a section of a bundle. In coordinates, this section should be defined by assigning to a point $q$ the Christoffel symbols at the point $q$ in the given chart. Note that this precludes the bundle whose sections are affine connections from being a vector bundle. We shall not carefully justify the assertions we make concerning the constructions in this section. However, they are fairly easily seen to make sense by simply considering the coordinate representations. The general constructions are described, for example, in [Kolář, Michor, and Slovák 1993].

The bundle of 1-jets of sections of $\pi_{\mathrm{TQ}}: \mathrm{TQ} \rightarrow \mathrm{Q}$ is denoted by $\mathrm{J}^{1}(\mathrm{TQ})$. This bundle is a vector bundle over $T Q$. We shall write a typical point in $J^{1}(T Q)$ in coordinates as $\left(q^{i}, X^{j}, X_{, \ell}^{k}\right)$. The notation $X_{, \ell}^{k}$ denotes the derivative of $X^{k}$ with respect to $q^{\ell}$. We denote by $\tau_{1}: \mathrm{J}^{1}(\mathrm{TQ}) \rightarrow \mathrm{TQ}$ the projection. In coordinates, $\tau_{1}$ has the form $\left(q^{i}, X^{j}, X_{, \ell}^{k}\right) \mapsto\left(q^{i}, X^{j}\right)$.

Next we have a canonical inclusion $\iota_{Q}$ of $\operatorname{End}(T Q)$ into $J^{1}(T Q)$ by asking that we assign to $A \in \operatorname{End}\left(\mathrm{T}_{q} \mathrm{Q}\right)$ the 1 -jet of any vector field with $q$ as an equilibrium point, and whose linearisation at $q$ is $A$. In coordinates, this inclusion looks like $\left(q^{i}, A_{\ell}^{k}\right) \mapsto\left(q^{i}, 0, A_{\ell}^{k}\right)$. If we use the natural identification $\operatorname{End}(\mathrm{TQ}) \simeq \mathrm{T}^{*} \mathrm{Q} \otimes \mathrm{TQ}$, then this inclusion maps $\boldsymbol{d} f(q) \otimes X(q)$ to the 1 -jet at $q$ of the vector field $f X$, where $f$ is a function vanishing at $q$. This characterisation extends to higher-order jet bundles. An affine connection $\nabla$ uniquely specifies, and is uniquely specified by, a splitting $S_{\nabla}: \mathrm{TQ} \rightarrow \mathrm{J}^{1}(\mathrm{TQ})$ of the exact sequence

$$
0 \longrightarrow \operatorname{End}(\mathrm{TQ}) \stackrel{\iota_{\mathrm{Q}}}{\longrightarrow} \mathrm{J}^{1}(\mathrm{TQ}) \stackrel{\tau_{1}}{\longrightarrow} \mathrm{TQ} \longrightarrow 0
$$


of vector bundles (it is clear from our coordinate expressions that image $\left(\iota_{\mathrm{Q}}\right)=\operatorname{ker}\left(\tau_{1}\right)$ ). Thus $S_{\nabla}$ is a vector bundle map that has the property that $\tau_{1} \circ S_{\nabla}=\mathrm{id} \mathrm{TQ}$. In coordinates, if $\Gamma_{j k}^{i}, i, j, k \in\{1, \ldots, n\}$, are the Christoffel symbols for the affine connection $\nabla$, then $S_{\nabla}$ is given by

$$
\left(q^{i}, X^{j}\right) \mapsto\left(q^{i}, X^{j}, \Gamma_{\ell m}^{k} X^{m}\right) .
$$

One readily verifies from our coordinate expressions that this map indeed splits the sequence (5.1).

Motivated by the preceding discussion, we let $\operatorname{Aff}(\mathrm{Q})$ be the set of vector bundle maps from $T Q$ to $J^{1}(T Q)$. By $\operatorname{Aff}_{0}(Q)$ we denote the subbundle defined by the torsion-free affine connections. If we fix an affine connection $\nabla^{0}$, then the vector bundle map from TQ to $\mathrm{J}^{1}(\mathrm{TQ})$ defined by $v_{q} \mapsto S_{\nabla}\left(v_{q}\right)-S_{\nabla^{0}}\left(v_{q}\right)$ is then uniquely defined by $D_{\nabla, \nabla^{0}} \in$ $\Gamma^{\infty}\left(\mathrm{T}^{*} \mathrm{Q} \otimes \mathrm{TS}^{2}(\mathrm{TQ})\right)$ satisfying $\nabla_{X} Y-\nabla_{X}^{0} Y=D_{\nabla, \nabla^{0}}(X, Y)$. In coordinates we shall denote a point in $\operatorname{Aff}(\mathrm{Q})$ by $\left(q^{i}, \Gamma_{k \ell}^{j}\right)$.

5.3. The partial differential equation of kinetic energy shaping. As we did with the potential shaping formulation, we must define a submanifold of the jet bundle of a suitable fibre bundle. We let $\Sigma_{2}^{+}(\mathrm{TQ})$ denote the set of Riemannian metrics on Q. Note that $\Sigma_{2}^{+}(\mathrm{TQ})$ is an open submanifold of the vector bundle $\mathrm{T}_{2}^{0}(\mathrm{TQ})$. The space of variables for our problem is the fibre bundle $\mathrm{ES}(\mathrm{Q}) \triangleq \Sigma_{2}^{+}(\mathrm{TQ}) \times(\mathrm{Gyr}(\mathrm{TQ}) \cap \operatorname{ker}(\mathrm{Alt}))$ over $\mathrm{Q}$. Following our declaration at the beginning of this section, we denote a typical point in the fibre of $\operatorname{ES}(\mathrm{Q})$ over $q \in \mathrm{Q}$ by $(\mathrm{G}(q), B(q))$. Note that the open-loop system defines a section $q \mapsto \mathrm{G}_{\mathrm{ol}}(q)$ of $\Sigma_{2}^{+}(\mathrm{TQ})$. We next let $\mathrm{J}^{1}(\mathrm{ES}(\mathrm{Q}))$ denote the bundle of 1-jets of sections of $\mathrm{ES}(\mathrm{Q})$. A typical point in $\mathrm{J}^{1}(\mathrm{ES}(\mathrm{Q}))$ will be denoted by $\left(j^{1} \mathrm{G}(q), j^{1} B(q)\right)$. In coordinates we shall write a typical point in $\mathrm{J}^{1}(\mathrm{ES}(\mathrm{Q}))$ as $\left(q^{i}, \mathrm{G}_{j k}, B_{l m r}, \mathrm{G}_{p s, a}\right)$, where $\mathbb{G}_{p s, a}$ represents the derivative of $\mathbb{G}_{p s}$ with respect to $q^{a}$. The Levi-Civita connection associated to a Riemannian metric $G$ can be thought of as being defined by a map from $J^{1}\left(\Sigma_{2}^{+}(T Q)\right)$ to $\operatorname{Aff}_{0}(Q)$. Let us denote this map by LC. In coordinates LC is given by

$$
\left(q^{i}, \mathbb{G}_{j k}, \mathbb{G}_{\ell m, r}\right) \mapsto\left(q^{i}, \mathbb{G}^{j r}\left(\mathbb{G}_{r k, \ell}+\mathbb{G}_{r \ell, k}-\mathbb{G}_{k \ell, r}\right)\right)
$$

To define the partial differential equation, we need some vector bundles adapted to the problem. To simplify the problem, we make the assumption that the codistribution $\mathcal{F}$ generated by the input one-forms is regular, so implying that the distribution $\mathbb{G}_{\mathrm{ol}}^{\sharp}(\mathcal{F})$ is also regular. This implies that the right ideal (with respect to the tensor product) in $\Gamma^{\infty}(\mathrm{T}(\mathrm{TQ}))$ generated by $\Gamma^{\infty}(\mathcal{F})$ is actually the set of sections of a subbundle of $\mathrm{T}(\mathrm{TQ})$. If $A$ is a $(0, k)$ tensor field and if $\mathbb{G}$ is a Riemannian metric, we denote by $\mathbb{G}^{\sharp} A$ the $(1, k-1)$-tensor field defined by

$$
\mathbb{G}^{\sharp} A\left(\alpha, X_{1}, \ldots, X_{k}\right)=A\left(\mathbb{G}^{\sharp}(\alpha), X_{1}, \ldots, X_{k}\right)
$$

where $\alpha \in \Gamma^{\infty}\left(\mathrm{T}^{*} \mathrm{Q}\right)$ and $X_{1}, \ldots, X_{k} \in \Gamma^{\infty}(\mathrm{TQ})$. In like fashion, if $\mathscr{S} \subseteq \mathrm{T}(\mathrm{TQ})$, then we write $\mathbb{G}(\mathscr{S})=\left\{\mathbb{G}^{\sharp} A \mid A \in \mathscr{S}\right\}$.

Now we are ready to define the fibred subset of $J^{1}(E S(Q))$ that will serve as our partial differential equation. We define

$$
\begin{aligned}
P_{\mathrm{KS}}\left(\mathbb{G}_{\mathrm{ol}}\right)_{q}=\left\{\left(j^{1} \mathrm{G}(q), j^{1} B(q)\right) \in \mathrm{J}^{1}(\mathrm{ES}(\mathrm{Q})) \mid\right. \\
\left(\mathrm{LC}\left(j^{1} \mathrm{G}(q)\right)-\mathrm{LC}\left(j^{1} \mathrm{G}_{\mathrm{ol}}(q)\right)+\mathbb{G}^{\sharp} B \in \mathbb{G}_{\mathrm{ol}}^{\sharp}\left(\mathcal{F} \otimes \mathrm{TS}^{2}(\mathrm{TQ})\right)\right\} .
\end{aligned}
$$


Note that $\mathrm{LC}\left(j^{1} \mathrm{G}(q)\right)-\mathrm{LC}\left(j^{1} \mathbb{G}_{\mathrm{ol}}(q)\right)$ is to be thought of as being a section of $\mathrm{T}^{*} \mathrm{Q} \otimes \mathrm{TS}{ }^{2}(\mathrm{TQ})$.

With all of this notation, we may now state the form of the partial differential equation associated with Problem 4.7.

5.2 Proposition: A Riemannian metric $\mathrm{G}_{\mathrm{cl}}$ and a gyroscopic tensor field $B_{\mathrm{cl}}$ solve Problem 4.7 if and only if the 1 -jet of the section $q \mapsto\left(\mathbb{G}_{\mathrm{cl}}(q), B_{\mathrm{cl}}(q)\right)$ takes values in $P_{\mathrm{KS}}\left(\mathbb{G}_{\mathrm{ol}}\right)$.

Proof: Note that the definitions, once parsed, assert that $\left(j^{1} \mathrm{G}_{\mathrm{cl}}, j^{1} B_{\mathrm{cl}}\right)$ takes values in $P_{\mathrm{KS}}\left(\mathrm{G}_{\mathrm{ol}}\right)$ if and only if there exists sections $F$ of $\mathcal{F} \otimes \mathrm{TS}^{2}(\mathrm{~V})$ and $B_{\mathrm{cl}}$ of $\operatorname{Gyr}(\mathrm{TQ}) \cap \operatorname{ker}(\mathrm{Alt})$ such that

$$
\stackrel{\mathrm{G}}{\mathrm{cl}}_{X} X(q)-\stackrel{\mathrm{G}}{\mathrm{ol}}_{X} X(q)=\mathbb{G}_{\mathrm{ol}}^{\sharp} F(X(q), X(q))-\mathrm{G}_{\mathrm{cl}}^{\sharp} B(X(q), X(q))
$$

for every $X \in \Gamma^{\infty}(\mathrm{TQ})$. This, however, exactly characterises a solution to Problem 4.7.

In coordinates, the partial differential equation is given by

$$
P_{r}^{a}\left(\mathbb{G}_{\mathrm{cl}}^{r \ell}\left(\mathbb{G}_{\mathrm{cl}, \ell j, k}+\mathbb{G}_{\mathrm{cl}, \ell k, j}-\mathbb{G}_{\mathrm{cl}, j j, \ell}\right)-\mathbb{G}_{\mathrm{ol}}^{r \ell}\left(\mathbb{G}_{\mathrm{ol}, \ell j, k}+\mathbb{G}_{\mathrm{ol}, \ell k, j}-\mathbb{G}_{\mathrm{ol}, j j, \ell}\right)+\mathbb{G}_{\mathrm{cl}}^{r \ell} B_{\ell j j}\right)=0,
$$

where $P_{r}^{a}, a \in\{1, \ldots, n-\operatorname{rank}(\mathcal{F})\}, r \in\{1, \ldots, m\}$, are the components of the canonical projection

$$
\pi_{\mathcal{F}}: \mathbb{G}_{\mathrm{ol}}^{\sharp}\left(\mathrm{T}^{*} \mathrm{Q} \otimes \mathrm{TS}^{2}(\mathrm{TQ})\right) \rightarrow \mathbb{G}_{\mathrm{ol}}^{\sharp}\left(\mathrm{T}^{*} \mathrm{Q} \otimes \mathrm{TS}^{2}(\mathrm{TQ})\right) / \mathbb{G}_{\mathrm{ol}}^{\sharp}\left(\mathcal{F} \otimes \mathrm{TS}^{2}(\mathrm{TQ})\right) .
$$

The advantage to the formulation of the problem as in Proposition 5.2 is that one can now apply the full power of the integrability theory of Goldschmidt [1967] for such systems. This is not to trivialise this process. However, it does give one a starting point.

\section{References}

Auckly, D. R. and Kapitanski, L. V. [2002] On the $\lambda$-equations for matching control laws, SIAM Journal on Control and Optimization, 41(5), pages 1372-1388, ISSN: 0363-0129, DOI: $10.1137 / \mathrm{S} 0363012901393304$.

Auckly, D. R., Kapitanski, L. V., and White, W. [2000] Control of nonlinear underactuated systems, Communications on Pure and Applied Mathematics, 53(3), pages 354-369, ISSN: 0010-3640, DOI: 10 . 1002/(SICI) 1097-0312(200003) 53:3<354::AID-CPA3>3. $0 . \mathrm{CO} ; 2-\mathrm{U}$.

Blankenstein, G., Ortega, R., and van der Schaft, A. J. [2002] The matching conditions of controlled Lagrangians and IDA-passivity based control, International Journal of Control, 75(9), pages 645-665, ISSN: 0020-7179, DOI: 10.1080/00207170210135939.

Bloch, A. M., Chang, D. E., Leonard, N. E., and Marsden, J. E. [2001] Controlled Lagrangians and the stabilization of mechanical systems. II. Potential shaping, Institute of Electrical and Electronics Engineers. Transactions on Automatic Control, 46(10), pages 1556-1571, ISSN: 0018-9286, DOI: 10.1109/9.956051.

Bloch, A. M., Krishnaprasad, P. S., Marsden, J. E., and Sánchez de Alvarez, G. [1992] Stabilization of rigid body dynamics by internal and external torques, Automatica, A Journal of IFAC, the International Federation of Automatic Control, 28(4), pages 745756, ISSN: 0005-1098, DOI: 10.1016/0005-1098(92) 90034-D. 
Bloch, A. M., Leonard, N. E., and Marsden, J. E. [2000] Controlled Lagrangians and the stabilization of mechanical systems. I. The first matching theorem, Institute of Electrical and Electronics Engineers. Transactions on Automatic Control, 45(12), pages 22532270, ISSN: 0018-9286, DOI: 10.1109/9.895562.

Bryant, R. L., Chern, S. S., Gardner, R. B., Goldschmidt, H. L., and Griffiths, P. A. [1991] Exterior Differential Systems, number 18 in Mathematical Sciences Research Institute Publications, Springer-Verlag: New York/Heidelberg/Berlin, ISBN: 978-1-4613-9716-8.

Bullo, F. and Lewis, A. D. [2004] Geometric Control of Mechanical Systems, Modeling, Analysis, and Design for Simple Mechanical Systems, number 49 in Texts in Applied Mathematics, Springer-Verlag: New York/Heidelberg/Berlin, ISBN: 978-0-387-22195-3.

Chang, D. E., Bloch, A. M., Leonard, N. E., Marsden, J. E., and Woolsey, C. A. [2002] The equivalence of controlled Lagrangian and controlled Hamiltonian systems, ESAIM. Control, Optimisation and Calculus of Variations, 8, pages 393-422, ISSN: 1292-8119, DOI: $0.1051 /$ cocv : 2002045.

Goldschmidt, H. L. [1967] Existence theorems for analytic linear partial differential equations, Annals of Mathematics. Second Series, 86(2), pages 246-270, ISSN: 0003-486X, DOI: $10.2307 / 1970689$.

Hamberg, J. [1999] General matching conditions in the theory of controlled Lagrangians, in Proceedings of the 38th IEEE Conference on Decision and Control, IEEE Conference on Decision and Control, (Phoenix, AZ, Dec. 1999), Institute of Electrical and Electronics Engineers, pages 2519-2523, DOI: 10.1109/CDC.1999.831306.

- [2000] Simplified conditions for matching and for generalized matching in the theory of controlled Lagrangians, in Proceedings of the 2000 American Control Conference, American Control Conference, (Chicago, IL, June 2000), Institute of Electrical and Electronics Engineers, pages 3918-3923, DOI: 10.1109/ACC.2000.876957.

Kolář, I., Michor, P. W., and Slovák, J. [1993] Natural Operations in Differential Geometry, Springer-Verlag: New York/Heidelberg/Berlin, ISBN: 978-3-540-56235-1.

Lewis, A. D. [1998] Affine connections and distributions with applications to nonholonomic mechanics, Reports on Mathematical Physics, 42(1/2), pages 135-164, ISSN: 0034-4877, DOI: $10.1016 / \mathrm{S} 0034-4877(98) 80008-6$.

Ortega, R., Spong, M. W., Gómez-Estern, F., and Blankenstein, G. [2002] Stabilization of a class of underactuated mechanical systems via interconnection and damping assignment, Institute of Electrical and Electronics Engineers. Transactions on Automatic Control, 47(8), pages 1218-1233, ISSN: 0018-9286, DOI: 10.1109/TAC.2002.800770.

Spencer, D. C. [1962a] Deformation of structures on manifolds defined by transitive, continuous pseudogroups. I. Infinitesimal deformations of structure, Annals of Mathematics. Second Series, 76(3), pages 306-398, ISSN: 0003-486X, DOI: 10.2307/1970277.

- [1962b] Deformation of structures on manifolds defined by transitive, continuous pseudogroups. II. Deformations of structure, Annals of Mathematics. Second Series, 76(2), pages 399-445, ISSN: 0003-486X, DOI: 10.2307/1970367.

Takegaki, M. and Arimoto, S. [1981] A new feedback method for dynamic control of manipulators, Transactions of the ASME. Series G. Journal of Dynamic Systems, Measurement, and Control, 103(2), pages 119-125, ISSN: 0022-0434, DOI: 10.1115/1.3139651.

van der Schaft, A. J. [1986] Stabilization of Hamiltonian systems, Nonlinear Analysis. Theory, Methods, and Applications, 10(10), pages 1021-1035, ISSN: 0362-546X, DOI: 10.1016/0362-546X (86) 90086-6. 
Zenkov, D. V. [2002] Matching and stabilization of linear mechanical systems, in Proceedings of 2002 International Symposium on Mathematical Theory of Networks and Systems, International Symposium on Mathematical Theory of Networks and Systems, (South Bend, IN, July 2002). 